\title{
Parâmetros genéticos para a produção de leite em caprinos das raças Saanen e Alpina ${ }^{1}$
}

\author{
Genetic parameters for milk yield in Saanen and Alpina breed goats
}

\author{
Natália Irano², Annaiza Braga Bignardi², Fernando Sebastián Baldi Rey ${ }^{3}$, Izabelle Auxiliadora Molina de \\ Almeida Teixeira ${ }^{4}$ e Lucia Galvão Albuquerque ${ }^{4 *}$
}

\begin{abstract}
Resumo - Objetivou-se com este trabalho estimar parâmetros genéticos para a produção de leite acumulada até os 305 dias (P305) de cabras das raças Saanen e Alpina. Foram utilizadas as duas primeiras parições de cabras pertencentes a rebanhos participantes do programa de controle produtivo e reprodutivo de caprinos (PROCAPRI) da UNESP-FCAV-Jaboticabal-SP. A P305 foi analisada por meio de modelos de repetibilidade e bicaracterísticas. Para verificar a influência dos efeitos fixos sobre a característica analisada foram realizadas análises preliminares, pelo método de quadrados mínimos. Os componentes de covariâncias foram estimados pelo método da máxima verossimilhança restrita (REML), utilizando o programa Wombat. A duração da lactação, a idade da cabra ao parto, o rebanho, o ano de parto e a estação de parto foram importantes fontes de variação para a P305. Não houve diferença significativa entre as raças estudadas. As estimativas de herdabilidade e repetibilidade para a P305, obtidas com o modelo de repetibilidade, foram de 0,29 e 0,36, respectivamente. As estimativas de herdabilidade, obtidas pelos modelos de repetibilidade e bicaracterísticas foram semelhantes. Sendo assim, um modelo de repetibilidade poderia ser indicado para avaliar a P305 pela sua simplicidade.
\end{abstract}

Palavras-chave - Caprinos-produção de leite. Genética animal. Leite de cabra.

\begin{abstract}
The objective of this study was to estimate genetic parameters for 305 days milk yield (M305) in Saanen and Alpina breed goats. Data consisted of records from first to second lactations of goats belonging to herds participating in the program control productive and reproductive goats (PROCAPRI) from UNESP-FCAV-Jaboticabal-SP. The M305 was analyzed by repeatability and bi-trait models. The influence of fixed effects on the traits was analyzed preliminary analysis by the method of least squares. Variance components were estimated by restricted maximum likelihood method (REML), using the package Wombat. The duration of lactation, age of dam at calving, the herd, calving year and season of birth were important sources of variation for the M305. There was no significant difference between the breeds. Heritability and repeatability estimates obtained by repeatability model were 0.29 and 0.36 , respectively. The repeatability estimate was 0.32 . The heritability estimates obtained by the bi-trait models were similar and repeatability. Thus, a repeatability model might be indicated for its simplicity.
\end{abstract}

Key words - Goat-milk production. Animal genetics. Goat milk.

\footnotetext{
* Autor para correspondência

Recebido para publicação 27/09/2010; aprovado em 26/10/2011

Parte do trabalho de conclusão de curso do primeiro autor, apresentado à Faculdade de Ciências Agrárias e Veterinárias/UNESP, pesquisa financiada pelo CNPq

${ }^{2}$ Programa de Pós-Graduação em Genética e Melhoramento Animal, Departamento de Zootecnia, FCAV/UNESP, Jaboticabal-SP, Brasil, 14.884-900, nattirano@hotmail.com, annaizabb@hotmail.com

${ }^{3}$ Departamento de Nutrição e Produção Animal, FMVZ/USP, Pirassununga-SP, Brasil, 13.635-900, fernandobaldiuy @ gmail.com

${ }^{4}$ Departamento de Zootecnia, FCAV/UNESP, Via de Acesso Prof. Paulo Donato Castellane, s/n, Jaboticabal-SP, Brasil, 14.884-900, izabelle@

fcav.unesp.br, lgalb@fcav.unesp.br
} 


\section{Introdução}

O leite de cabra é um alimento de rico valor nutricional e baixa alergenicidade quando comparado ao leite bovino, por isso a procura no mercado por este produto é cada vez mais frequente. Segundo Guimarães et al. (2004), a crescente demanda por produtos derivados do leite de cabra, como leite em embalagem longa vida, leite em pó, queijos, iogurtes, achocolatado, entre outros, tem levado à melhoria do produto final.

Grande parte da variação existente na produção de leite é influenciada por fatores de ambiente como duração da lactação, ano e estação do parto, idade da cabra ao parto, ordem do parto, tipo de parto, raça, entre outros. De acordo com Soares Filho et al. (2001), a persistência prolongada da lactação resultará em diminuição na eficiência reprodutiva do animal ao longo de sua vida produtiva. Pimenta Filho et al. (2004) relataram que a duração da lactação influencia a produção de leite de forma quadrática e que lactações mais longas não necessariamente resultarão em uma maior produção de leite.

Segundo Gonçalves et al. (2001), o efeito da estação do parto poderia ser explicado pelas alterações climáticas, as quais afetam os animais direta ou indiretamente. Estes autores também relataram que cabras de primeiro parto produzem menos leite que as adultas, devido ao incompleto desenvolvimento corporal e fisiológico que as cabras mais jovens apresentam. Soares Filho et al. (2001) observaram influência significativa da raça sobre a produção de leite, sendo que a raça Saanen apresentou superioridade na produção total de leite sobre a raça Alpina, a Toggenburg e seus mestiços. Por outro lado, Guimarães (2004) relatou que não houve diferença para a produção de leite quando se compararam animais das raças Saanen e Alpina, criadas em mesmas condições de alimentação e manejo.

As análises de dados da produção de leite durante a vida da cabra podem ser conduzidas de várias formas. Podem ser analisadas considerando-se a produção acumulada aos 305 dias (P305) por meio de modelos de repetibilidade ou multicaracterísticas. O modelo de repetibilidade, com menor complexidade em relação à análise multicaracterísticas, assume que medidas tomadas em diferentes idades, em um mesmo animal, representam repetições de uma mesma característica, considerando correlação genética entre as produções igual a unidade e variâncias genéticas e não genéticas constantes. Já nos modelos multicaracterísticas é assumido que as produções totais de diferentes lactações representam características diferentes, sendo que as covariâncias e correlações genéticas entre lactações diferentes variam, mas nenhuma pressuposição é feita sobre a estrutura de covariâncias. Com estes modelos, o número de parâmetros a ser estimado aumenta em forma exponencial à medida que a quantidade de características na análise é maior (MEYER, 1997).

No Brasil, são escassas as estimativas de herdabilidade para a característica P305 em caprinos. Ribeiro (1997), utilizando dados de cabras da raça Saanen e, Soares Filho et al. (2001), trabalhando com cabras das raças Saanen, Alpina e Toggenburg, obtiveram estimativas de herdabilidade para produção de leite que variaram de 0,06 a 0,12. Já Tholon et al. (2001), trabalhando com cabras da raça Saanen e, Guimarães (2004), estudando cabras das raças Saanen e Alpina, estimaram herdabilidades maiores, 0,37 e 0,47 respectivamente.

Objetivou-se com este trabalho estimar parâmetros genéticos para a produção de leite acumulada até os 305 dias de cabras das raças Saanen e Alpina.

\section{Material e métodos}

Foram utilizadas 988 lactações incluindo as duas primeiras parições de 495 cabras das raças Saanen e 319 cabras da raça Alpina, filhas de 128 bodes. Os animais são provenientes de 24 rebanhos, a maioria da região Sudeste do Brasil (Estados de São Paulo e Minas Gerais), participantes do Programa de controle produtivo e reprodutivo de caprinos (PROCAPRI) da UNESPFCAV-Jaboticabal-SP, no período de 1999 a 2004. Os animais apresentaram, em média, 1,2 lactações.

A maioria dos animais foi criada em regime intensivo para produção de leite, conforme recomendações do NRC (1981). Em termos gerais, a alimentação era baseada em silagem de milho como volumoso principal e concentrado à base de grão de milho, farelo de soja, soja tostada e calcário. A silagem de milho, o sal mineral e a água foram fornecidos à vontade e o concentrado foi fornecido na ordenha da manhã. Uma pequena parte dos animais foi criada sob sistema de criação de semi-confinamento, onde os animais tinham acesso ao pasto por algumas horas. $\mathrm{O}$ manejo geral dos rebanhos incluiu o acompanhamento e controle de ecto e endoparasitas. O controle preventivo de mastite foi feito por meio de uso de caneca telada, lavagem das tetas antes da ordenha e posterior imersão em iodo glicerinado. Foram feitas duas ordenhas diárias e colhidos os dados das lactações.

A característica analisada foi a produção de leite acumulada até 305 dias (P305) para as duas primeiras lactações. As lactações foram truncadas aos 305 dias e foram eliminadas cabras com idade ao primeiro parto inferior a 365 dias e lactações com duração menor que 90 dias. 
Para verificar a influência dos efeitos fixos sobre a característica analisadaforam realizadas análises preliminares, pelo método de quadrados mínimos. Foram considerados os efeitos fixos de rebanho, ano de parto, estação de parto, raça, idade da cabra ao parto e duração da lactação. O grupo de contemporâneos foi definido como rebanho-ano-estação do parto. De acordo com a concentração dos partos, foram determinadas duas estações de parição, a primeira nas águas, entre os meses de outubro a março e, a segunda na seca, entre os meses de abril a setembro. Aplicou-se a restrição de que cada grupo de contemporâneos deveria conter, no mínimo, três observações.

A P305 foi analisada por meio de modelos de repetibilidade e bicaracterísticas, considerando as duas primeiras lactações. $\mathrm{O}$ modelo de repetibilidade incluiu o grupo de contemporâneos como efeito fixo, a duração da lactação (regressão linear) e a idade da cabra ao parto (regressão linear e quadrática) como covariáveis e, os efeitos, genético aditivo e de ambiente permanente como aleatórios. Já o modelo bicaracterísticas, incluiu os mesmos efeitos que no modelo de repetibilidade com exceção do efeito de ambiente permanente do animal. Em todas as análises foi utilizado um arquivo de pedigree, com 2.793 animais na matriz de parentesco.

O modelo geral pode ser representado na forma matricial como demonstarado na equação 1 :

$\mathrm{y}=\mathrm{X} \beta+\mathrm{Za}+\mathrm{Wc}+\mathrm{e}$

Em que:

$\mathbf{y}=$ vetor da variável dependente;

$\mathbf{X}=$ matriz de incidência de efeitos fixos para a variável dependente;

$\beta=$ vetor de efeitos fixos;

$\mathbf{Z}=$ matriz de incidência de efeitos genéticos aditivos diretos;

$\mathbf{a}=$ vetor de efeitos genéticos aditivos diretos;

$\mathbf{W}=$ matriz de incidência do efeito ambiente permanente;

$\mathbf{c}=$ vetor do efeito de ambiente permanente;

$\mathbf{e}=$ vetor de efeitos aleatórios residuais associados às observações.
Os componentes de covariâncias, necessários à estimação dos parâmetros genéticos, foram estimados pelo método da máxima verossimilhança restrita (REML), utilizando o programa Wombat (MEYER, 2006).

\section{Resultados e discussão}

As médias observadas, os desvios-padrão e os coeficientes de variação $(\mathrm{CV} \%)$ para a produção de leite até 305 dias encontram-se na Tabela 1. A média de produção de leite estimada neste trabalho foi menor que as encontradas por Gonçalves et al. (2001), Tholon et al. (2001), Soares Filho et al. (2001), e Guimarães (2004), os quais relataram valores que variaram de 569,32 a 766,4 kg, em cabras das raças Alpina, Saanen e Toggenburg. Valor inferior para a produção total de leite foi observado por Pimenta Filho et al. (2004), que relataram média de $257,7 \pm 54,7 \mathrm{~kg}$ para cabras mestiças. A produção média de leite aumentou da primeira para a segunda lactação. Os coeficientes de variação para a produção de leite foram altos, provavelmente, devido à variação que existe entre os diferentes rebanhos estudados, apesar do sistema de criação e manejo alimentar ser semelhante entre os rebanhos.

A produção média diária foi de $2,4 \mathrm{~kg}$, valores similares aos reportados em outros trabalhos estudando a produção de leite de cabras da raça Saanen e Alpina (SARMENTO et al., 2006; THOLON et al., 2001). A média para duração da lactação foi menor que a encontrada por Soares Filho et al. (2001) e Lôbo e Silva (2005), que foram 238,43 e 239,13 dias respectivamente, em cabras da raça Saanen.

Os resultados da análise de variâncias para a P305 pode ser observada na Tabela 2. Os efeitos fixos considerados no modelo foram responsáveis por $81,2 \%$ da variação total da produção de leite. Não houve diferença significativa $(\operatorname{Pr}>0,05)$ entre as raças para a P305. Sendo assim, pode-se sugerir que as raças Saanen e Alpina, nos rebanhos avaliados, apresentam potencial semelhante para a produção de leite. Isto pode ser explicado pelo fato de que as duas raças estudadas têm mesma origem (Suécia).

Tabela 1 - Número de observações, médias, desvios-padrão (DP) e coeficientes de variação (CV) para a produção de leite acumulada até 305 dias, duração da lactação (DL) e idade da cabra ao parto (IDCP)

\begin{tabular}{|c|c|c|c|c|c|c|c|c|}
\hline \multirow[t]{2}{*}{ Características } & \multirow[t]{2}{*}{$\mathrm{N}^{\circ}$ de observações } & \multicolumn{3}{|c|}{ Produção de leite $(\mathrm{kg})$} & \multicolumn{2}{|c|}{ DL } & \multicolumn{2}{|c|}{ IDCP (meses) } \\
\hline & & Média & DP & $\mathrm{CV}(\%)$ & Média & $\mathrm{DP}$ & Média & DP \\
\hline Todas as lactações & 988 & 511,70 & 351,67 & 69 & 211,40 & 73,20 & 26,05 & 12,39 \\
\hline $1^{\mathrm{a}}$ lactação & 649 & 495,77 & 347,00 & 70 & 213,00 & 74,70 & 21,72 & 10,63 \\
\hline $2^{\mathrm{a}}$ lactação & 339 & 542,20 & 358,99 & 66 & 208,33 & 70,22 & 34,35 & 11,25 \\
\hline
\end{tabular}


Guimarães (2004), comparando animais das raças Saanen e Alpina, também não observou diferença significativa para este efeito.

Tabela 2 - Análise de variâncias para a produção de leite acumulada até 305 dias

\begin{tabular}{lccl}
\hline Fonte de variação & GL & Valor de F & Pr > F \\
\hline Rebanho & 24 & 65,82 & $0,0001^{*}$ \\
Ano de parto & 11 & 4,94 & $0,0001^{*}$ \\
Estação de Parto & 1 & 4,23 & $0,0400^{*}$ \\
Raça & 1 & 0,84 & 0,3596 \\
IDCP (linear) & 1 & 66,75 & $0,0001^{*}$ \\
IDCP (quadrático) & 1 & 62,30 & $0,0001^{*}$ \\
DL (linear) & 1 & 11,56 & $0,0007^{*}$ \\
DL (quadrático) & 1 & 0,41 & 0,5210 \\
\hline
\end{tabular}

* Significativo ao nível de 5\%, IDCP = idade da cabra ao parto e DL = duração da lactação

A duração da lactação (DL) afetou de forma significativa a produção de leite, verificando-se apenas efeito linear para esta característica. A cada dia que a duração da lactação se estende, a produção total acumulada de leite aumenta $2,02 \mathrm{~kg}(\mathrm{Y}=83,11+2,02 * \mathrm{DL})$. O efeito linear da duração da lactação sobre a produção de leite também foi observado por Ribeiro (1997), Tholon et al. (2001) e Guimarães (2004). O efeito quadrático da duração da lactação não influenciou significativamente $(\operatorname{Pr}>0.05)$ a produção de leite. Entretanto, Pimenta Filho et al. (2004) observaram efeito quadrático da duração da lactação sobre a produção de leite para cabras mestiças.

Os efeitos linear e quadrático da idade da cabra ao parto (IDCP) influenciaram significativamente a produção de leite $\left(\mathrm{Y}=712,34-0,34 * \mathrm{IDCP}+0,01 * \mathrm{IDCP}^{2}\right)$. Esse resultado é biologicamente esperado, uma vez que a cabra aumenta sua produção de leite até certa idade e tende a decrescer, descrevendo assim uma função quadrática, o que corrobora com a maioria dos trabalhos encontrados na literatura sobre caprinos (GUIMARÃES, 2004; GONÇALVES et al., 2001; MOURAD, 1992; PIMENTA FILHO et al., 2004; SOARES FILHO et al., 2001). O efeito da idade da cabra ao parto sobre a característica estudada, provavelmente foi bem evidenciado neste estudo, devido à existência no rebanho tanto de cabras jovens como de cabras maduras.

Como já era esperado, os efeitos sistemáticos do ambiente, como rebanho, ano e estação de parto, foram uma importante fonte de variação para a P305 e, devem portanto, ser considerados no modelo. Uma correta definição dos grupos de contemporâneos é um dos aspectos mais importantes para predizer valores genéticos confiáveis para qualquer característica, pois grande parte dos efeitos ambientais e suas interações são consideradas nestes grupos.

As estimativas dos componentes de variância e dos coeficientes de herdabilidade e repetibilidade, para a P305, podem ser observadas na Tabela 3. As estimativas das variâncias residuais e fenotípicas obtidas pelo modelo bicaracterísticas mostraram a mesma tendência que as médias de produção de leite, aumentando da primeira para a segunda lactação. Já a estimativa de variância genética diminui da primeira para a segunda lactação, indicando um decréscimo da variabilidade genética para a P305 na segunda lactação. Para a primeira lactação, a estimativa de variância genética obtida pelo modelo de repetibilidade, foi próxima à estimada pelo modelo bicaracterísticas. Entretanto, a estimativa de variância genética para a segunda lactação, obtida pelo modelo bicaracterísticas, foi menor que aquela obtida pelo modelo de repetibilidade. Provavelmente, o menor número de informações na segunda lactação ocasiona diferença entre os modelos na partição da variância genética. Para os dois modelos, os altos valores das estimativas de variâncias residuais, sugerem a existência de fatores do meio ambiente, de manejo e genéticos (dominância e/ou epistasia), que não foram considerados no modelo (não identificáveis), mas que influenciam na produção de leite.

A estimativa do coeficiente de repetibilidade para P305 pode ser considerada de moderada magnitude, o que indica a possibilidade de utilização das primeiras produções de leite das cabras como indicativo de sua produção futura, selecionando, assim, as fêmeas que continuarão produzindo no rebanho. Entretanto, devido ao moderado coeficiente de repetibilidade encontrado neste trabalho, maior número de informações é necessário (mais de uma lactação por cabra) para se tomar decisões precisas quanto ao descarte dos animais. Entretanto, isso provocaria aumento nos custos de produção, pois animais de baixa produção teriam que permanecer por mais tempo no rebanho e, consequentemente, diminuição do lucro. Estimativas de repetibilidade inferiores às obtidas neste estudo foram relatadas por Ribeiro (1997), Gonçalves et al. (2002) e Lôbo e Silva (2005) as quais variaram de 0,11 a 0,20. Por outro lado, Tholon et al. (2001), Mourad (2001) e Guimarães (2004) estimaram valores de repetibilidade superiores às obtidas no presente trabalho, sendo de 0,$52 ; 0,73$ e 0,61 , respectivamente.

As estimativas de herdabilidade para a P305, obtidas pelos modelos de repetibilidade e bicaracterísticas, foram moderadas. Estas estimativas sugerem que a P305 deve responder à seleção. Apesar das pressuposições 
Tabela 3 - Estimativas de variâncias genética aditiva $\left(\sigma_{\mathrm{a}}^{2}\right)$, residual $\left(\sigma_{\mathrm{e}}^{2}\right)$ e fenotípica $\left(\sigma_{\mathrm{p}}^{2}\right)$ e, dos coeficientes de herdabilidade $\left(\mathrm{h}^{2}\right)$ e repetibilidade (t) obtidas utilizando modelos de repetibilidade e bicaracterísticas $\left(1^{\mathrm{a}}\right.$ e $2^{\mathrm{a}}$ lactação)

\begin{tabular}{lccccc}
\hline \multicolumn{1}{c}{ Características } & $\sigma^{2}{ }_{\mathrm{a}}$ & $\sigma_{\mathrm{e}}^{2}$ & $\sigma_{\mathrm{p}}^{2}$ & $\mathrm{~h}^{2}$ & $\mathrm{t}$ \\
\hline Modelo repetibilidade & 5.445 & 11.930 & 18.677 & 0,29 & 0,36 \\
$1^{\mathrm{a}}$ lactação & 5.703 & 11.063 & 16.766 & 0,34 & - \\
$2^{\mathrm{a}}$ lactação & 3.717 & 16.109 & 19.827 & 0,19 & - \\
\hline
\end{tabular}

diferentes de cada modelo, as estimativas de componentes de variâncias e herdabilidades, obtidas pelos modelos de repetibilidade e bicaracterísticas, foram semelhantes para a primeira lactação.

A estimativa de herdabilidade para P305 obtida pelo modelo de repetibilidade é superior às estimativas relatadas por Gonçalves et al. (2002), Pimenta Filho et al. (2004) e Lôbo e Silva (2005), os quais também utilizaram um modelo de repetibilidade. Entretanto, Tholon et al. (2001), Silva et al. (2002) e Guimarães (2004), também empregando um modelo de repetibilidade, relataram estimativas de herdabilidade superiores, sendo 0,37; 0,33 e 0,47 respectivamente. As herdabilidades estimadas neste estudo para produção total na lactação (repetibilidade e bicaracterísticas) foram menores que as estimadas para as produções de leite no dia do controle de primeiras lactações de cabras, utilizando diferentes modelos, encontradas na literatura (BREDA et al., 2006; SARMENTO et al., 2006; SARMENTO et al., 2008).

A estimativa de correlação genética entre a primeira e a segunda lactação foi próxima a unidade $(0,99)$. Este resultado sugere que a produção de leite em cada lactação deve ser considerada como uma mesma característica. Em trabalhos com bovinos leiteiros, altas correlações genéticas têm sido estimadas entre produções de leite em diferentes lactações (ALBUQUERQUE et al., 1996; FREITAS et al., 2001; MEYER, 1984). Nestas condições, devido à proximidade das estimativas dos componentes de (co) variâncias e herdabilidades para as produções de leite nas duas primeiras lactações obtidas pelos dois modelos avaliados, um modelo de repetibilidade poderia ser indicado pela sua simplicidade para avaliar a P305 em cabras.

\section{Conclusões}

1. Os efeitos significativos das variáveis rebanho, ano de parto, estação de parto, idade da cabra ao parto e duração da lactação, indicam que estes devem ser considerados em estudos de estimativas de parâmetros genéticos para produção de leite em cabras;
2. Em avaliações genéticas para a característica produção de leite em cabras, o emprego do modelo de repetibilidade pode ser indicado por apresentar menor complexidade em relação ao modelo bicaracterísticas e possuir melhor ajuste dos dados;

3. As estimativas de herdabilidade sugerem que esta característica deve responder à seleção.

\section{Agradecimentos}

Ao Conselho Nacional de Desenvolvimento Científico e Tecnológico (CNPq), pelo apoio financeiro.

\section{Referências}

ALBUQUERQUE, L. G.; KEOWN, J. F.; VLECK, L. D. $\mathrm{V}$. Genetic parameters of milk, fat, and protein yields in the first three lactations, using an animal model and restricted maximum likelihood. Brazilian Journal of Genetics, v. 19, n. 01 , p. 79-86, 1996

BREDA, F. C. et al. Estimação de parâmetros genéticos para produção de leite de cabras da raça Alpina. Revista Brasileira de Zootecnia, v. 35, n. 02, p. 396-404, 2006.

FREITAS, A. F. et al. Parâmetros genéticos para produções de leite e gordura nas três primeiras lactações de vacas holandesas. Revista Brasileira de Zootecnia, v. 30, n. 03, p. 709-713, 2001.

GONÇALVES, H. C. et al. Fatores genéticos e de meio na produção de leite de caprinos leiteiros. Revista Brasileira de Zootecnia, v. 30, n. 03, p.719-729, 2001.

GONÇALVES, H. C. et al. Parâmetros e tendência genética da produção de leite de cabra no Brasil. Revista Brasileira de Zootecnia, v. 31, n. 06, p. 2204-2208, 2002.

GUIMARÃES, V. P. Curva de lactação, efeitos ambientais e genéticos sobre o desempenho produtivo de cabras leiteiras. 2004. 87 f. Dissertação (Mestrado em Zootecnia) - Universidade Federal de Viçosa, Viçosa.

LÔBO, R. N. B.; SILVA, F. L. R. Parâmetros genéticos para características de interesse econômico em cabras das raças Saanen e Anglo-nubiana. Revista Ciência Agronômica, v. 36, n. 01, p. 104-110, 2005. 
MEYER, K. Estimates of genetic parameters for milk and fat yield for first three lactations in British Friesian cows. Animal Production, v. 38, p. 313-322, 1984.

MEYER, K. Estimates of genetic parameters for weaning weight of beef cattle accounting for direct-maternal covariances. Livestock Production Science, v. 52, p. 187199, 1997.

MEYER, K. WOMBAT. Digging deep for quantitative genetic analyses by restricted maximum likelihood. In: WORLD CONGRESS ON GENETICS APPLIED TO LIVESTOCK PRODUCTION, 8., 2006, Belo Horizonte. Proceedings... Belo Horizonte. 1 CD-ROM.

MOURAD, M. Effects of month of kidding, parity and litter size on milk yield of Alpine goats in Egypt. Small Ruminants Research, v. 08, n. 01/02, p. 41-46, 1992.

MOURAD, M. Estimation of repeatability of milk yield and reproductive traits of Alpine goats under an intensive system of production in Egypt. Small Ruminant Research, v. 42, n. 01, p. 1-4, 2001.

PIMENTA FILHO, E. C.; SARMENTO, J. L. R.; RIBEIRO, M. N. Efeitos genéticos e ambientais que afetam a produção de leite e duração da lactação de cabras mestiças no Estado da Paraíba. Revista Brasileira de Zootecnia, v. 33, n. 06, p. 1426-1431, 2004.
RIBEIRO, A. C. Estudo dos efeitos genéticos e de ambiente sobre características de importância econômica em caprinos da raça Saanen. 1997. 116 f. Dissertação (Mestrado em Melhoramento Genético Animal) - Faculdade de Ciências Agrárias e Veterinárias, Universidade Estadual Paulista, Jaboticabal.

SARMENTO, J. L. R. et al. Avaliação genética de caprinos da raça Alpina utilizando-se a produção de leite no dia do controle. Revista Brasileira de Zootecnia, v. 35, n. 02, p. 443-451, 2006.

SARMENTO, J. L. R. et al. Comparação de modelos de regressão aleatória para estimação de parâmetros genéticos em caprinos leiteiros. Revista Brasileira de Zootecnia, v. 37, n. 10, p. 1788-1796, 2008.

SILVA, F. L. R.; ANDRADE, V. O.; LIMA, F. A. M. Estimativas de herdabilidade para produção de leite em cabras mestiças no semi-árido do Nordeste do Brasil. Revista Científica de Produção Animal, v. 04, n. 01-02, p. 64-07, 2002.

SOARES FILHO, G.; MCMANUS, C.; MARIANTE, A. $\mathrm{S}$. Fatores genéticos e ambientais que influenciam algumas características de reprodução e produção de leite em cabras no Distrito Federal. Revista Brasileira de Zootecnia, v. 30, n. 01, p. 133-140, 2001

THOLON, P. et al. Estudo genético quantitativo da produção de leite em caprinos da raça Saanen. Archivos Latinoamericanos Producción Animal, v. 09, n. 01, p. 1-5, 2001. 